\title{
Bilateral adrenal masses due to tuberculosis: how to diagnose without extra-adrenal tuberculosis
}

\author{
Nam Quang Tran1,2, Chien Cong Phan33, Thao Thi Phuong Doan ${ }^{4}$ and Thang Viet Tran 1,2 \\ 'Department of Endocrinology, University of Medicine and Pharmacy at Ho Chi Minh City, Ho Chi Minh City, Vietnam, \\ 2Department of Endocrinology, ${ }^{3}$ Department of Imaging, University Medical Center at Ho Chi Minh City, Ho Chi Minh \\ City, Vietnam, and ${ }^{4}$ Department of Histopathology, University of Medicine and Pharmacy at Ho Chi Minh City, Ho Chi \\ Minh City, Vietnam
}

Correspondence should be addressed to N Q Tran

Email

nam.tq@umc.edu.vn

\section{Summary}

Primary adrenal insufficiency is a rare disease and can masquerade as other conditions; therefore, it is sometimes incorrectly diagnosed. Herein, we reported the case of a 39-year-old Vietnamese male with primary adrenal insufficiency due to bilateral adrenal tuberculosis. The patient presented to the emergency room with acute adrenal crisis and a 3-day history of nausea, vomiting, epigastric pain, and diarrhoea with a background of 6 months of fatigue, weight loss, and anorexia. Abdominal CT revealed bilateral adrenal masses. Biochemically, unequivocal low morning plasma cortisol (<83 nmol/L) and high plasma adrenocorticotropic hormone levels were consistent with primary adrenal insufficiency. There was no evidence of malignancy or lymphoma. As the patient was from a tuberculosis-endemic area, extra-adrenal tuberculosis was excluded during the work up. A retroperitoneal laparoscopic left adrenalectomy was performed, and tuberculous adrenalitis was confirmed by the histopathological results. The patient was started on antituberculous therapy, in addition to glucocorticoid replacement. In conclusion, even without evidence of extra-adrenal tuberculosis, a diagnosis of bilateral adrenal tuberculosis is required. A histopathological examination has a significant role along with clinical judgement and hormonal workup in establishing a definitive diagnosis of adrenal tuberculosis without evidence of active extra-adrenal involvement.

\section{Learning points:}

- Primary adrenal insufficiency can be misdiagnosed as other mimicking diseases, such as gastrointestinal illness, leading to diagnostic pitfalls.

- Adrenal insufficiency can be confirmed with significantly low morning plasma cortisol levels of $<83 \mathrm{nmol} / \mathrm{L}$ without a dynamic short cosyntropin stimulation test.

- Tuberculous adrenalitis is an uncommon treatable condition; however, it remains an important cause of primary adrenal insufficiency, especially in developing countries. In the absence of extra-adrenal involvement, adrenal biopsy plays a key role in the diagnostic process. Alternatively, adrenalectomy for histopathological purposes should be considered if CT scan-guided fine needle aspiration is infeasible in cases of small adrenal masses.

\section{Background}

In the developing countries, tuberculosis continues to be heavy burden of tuberculosis in the world. More than $80 \%$ of the primary adrenal insufficiency cases in the developed world are caused by autoimmune diseases (gradual destruction of adrenal cortex by cytotoxic lymphocytes) $(1,2,3)$. However, in developing countries, tuberculosis 
of the adrenal gland is an important aetiology in primary adrenal insufficiency $(3,4)$.

Primary adrenal insufficiency is a rare condition which is associated with significant morbidity and mortality. The clinical characteristics of adrenal insufficiency depend on its rate of onset and severity. Diagnosis is occasionally established when the patient is in an adrenal crisis. The most frequent manifestations of cortisol deficiency in primary adrenal insufficiency are vague, nonspecific, and gradually progressing such as fatigue, weakness, anorexia, weight loss, nausea, vomiting, and postural hypotension; thus, primary adrenal insufficiency can be easily misdiagnosed as other mimicking disorders. Skin hyperpigmentation is a recognised feature that helps to differentiate primary from secondary hypoadrenalism, which is present in up to $94 \%$ of the cases (3). Bilateral adrenal masses due to tuberculosis can be clinically diagnosed if evidence of tuberculosis is also found in other organs (e.g. lungs); otherwise, confirmation of adrenal tuberculosis becomes challenging. Approximately $25 \%$ of the patients with tuberculous adrenalitis have no active extra-adrenal involvement (5).

Herein, we reported a case of bilateral tuberculous adrenalitis, wherein the patient initially presented with gastrointestinal symptoms, bilateral adrenal masses, and no evidence of extra-adrenal tuberculosis.

\section{Case presentation}

A previously healthy, nonsmoker, 39-year-old Vietnamese male presented to the gastroenterology outpatient clinic with a 3-day history of worsening recurrent nausea, vomiting, diarrhoea, and stomach upset. He reported experiencing weakness, anorexia, $10 \mathrm{~kg}$ of unintentional weight loss, and apparent skin hyperpigmentation over his face and hands in the past 6 months. He was diagnosed with gastritis. There was no history of lung tuberculosis, autoimmune diseases, head tumour, or brain irradiation. The patient had not been on any glucocorticoid products. No one in his family had primary adrenal insufficiency. In the outpatient clinic, he suddenly experienced acute abdominal pain and was immediately brought to the emergency department in a haemodynamically unstable condition.

He was hypotensive and tachycardic with a blood pressure of $90 / 60 \mathrm{mmHg}$ and a heart rate of 100 b.p.m. Skin pigmentation was obvious over his hands, nails, and the oral mucosal membrane. Painless bilateral small supraclavicular palpable lymph nodes were also observed. Other systemic examinations were unremarkable. An urgent abdominal CT scan was performed, which revealed prominent bilateral adrenal masses. Subsequently, he was admitted to the endocrinology ward for further work up and management.

\section{Investigation}

Adrenal investigations demonstrated significantly low morning plasma cortisol of $68.4 \mathrm{nmol} / \mathrm{L}$ which was consistent with impaired cortisol production by the adrenal glands. Concurrently, high plasma adrenocorticotropic hormone (ACTH) of $259.9 \mathrm{pmol} / \mathrm{L}$ (reference range, 1.6-13.9 pmol/L) indicated adrenal cortex destruction resulting in primary adrenal insufficiency. Additionally, high plasma renin concentration of $273.17 \mathrm{ng} / \mathrm{L}$ (reference range, 7.54-42.3 $\mathrm{ng} / \mathrm{L}$ ) and normal plasma aldosterone of $5.03 \mathrm{ng} / \mathrm{dL}$ (reference range, $2.5-31.5 \mathrm{ng} / \mathrm{dL}$ ) suggested mineralocorticoid deficiency. The routine biochemical profile also showed low blood glucose with no symptoms, mild hyponatremia, normal complete blood count, and normal thyroid function. Normal plasma metanephrines made pheochromocytomas unlikely (Table 1).

Abdominal CT revealed bilateral adrenal masses with the left mass measuring $20 \mathrm{~mm}$ and $30 \mathrm{HU}$ and the right measuring $15 \mathrm{~mm}$ (Fig. 1). There was also a 10-mm-thickened section in the ascending colon, suggestive of inflammation. Hence, a colonoscopy was performed, with subsequent normal findings.

The differential aetiologies of primary adrenal insufficiency with bilateral adrenal masses should be

Table 1 Blood tests and hormonal results of the patient.

\begin{tabular}{|c|c|c|}
\hline Laboratory findings & Patient & Reference range \\
\hline \multicolumn{3}{|l|}{ Blood tests } \\
\hline $\begin{array}{l}\text { Random plasma glucose } \\
\text { (mmol/L) }\end{array}$ & 3 & \\
\hline Sodium (mmol/L) & 132 & $136-146$ \\
\hline Potassium (mmol/L) & 4.79 & $3.4-5.1$ \\
\hline Gamma interferon (pg/mL) & 3.75 & $<25$ \\
\hline \multicolumn{3}{|l|}{ Serume hormones } \\
\hline $\begin{array}{l}\text { Morning plasma cortisol } \\
\text { (nmol/L) }\end{array}$ & 68.4 & $118.6-618$ \\
\hline ACTH (pmol/L) & 259.9 & 1.6-13.9 \\
\hline DHEA-S (pg/mL) & 0.173 & $0.69-6.17$ \\
\hline FT4 (pmol/L) & 9.85 & 9.0-19.0 \\
\hline $\mathrm{TSH}(\mathrm{mUI} / \mathrm{L})$ & 2.43 & $0.27-4.2$ \\
\hline Plasma aldosterone (ng/dL) & 5.03 & $2.5-31.5$ \\
\hline Direct renin concentration (ng/L) & 273.17 & $7.54-42.3$ \\
\hline Plasma metanephrines (pg/mL) & 10.14 & $<90$ \\
\hline
\end{tabular}

ACTH, adrenocorticotropic hormone; FT4, free T4; TSH, thyroidstimulating hormone. 

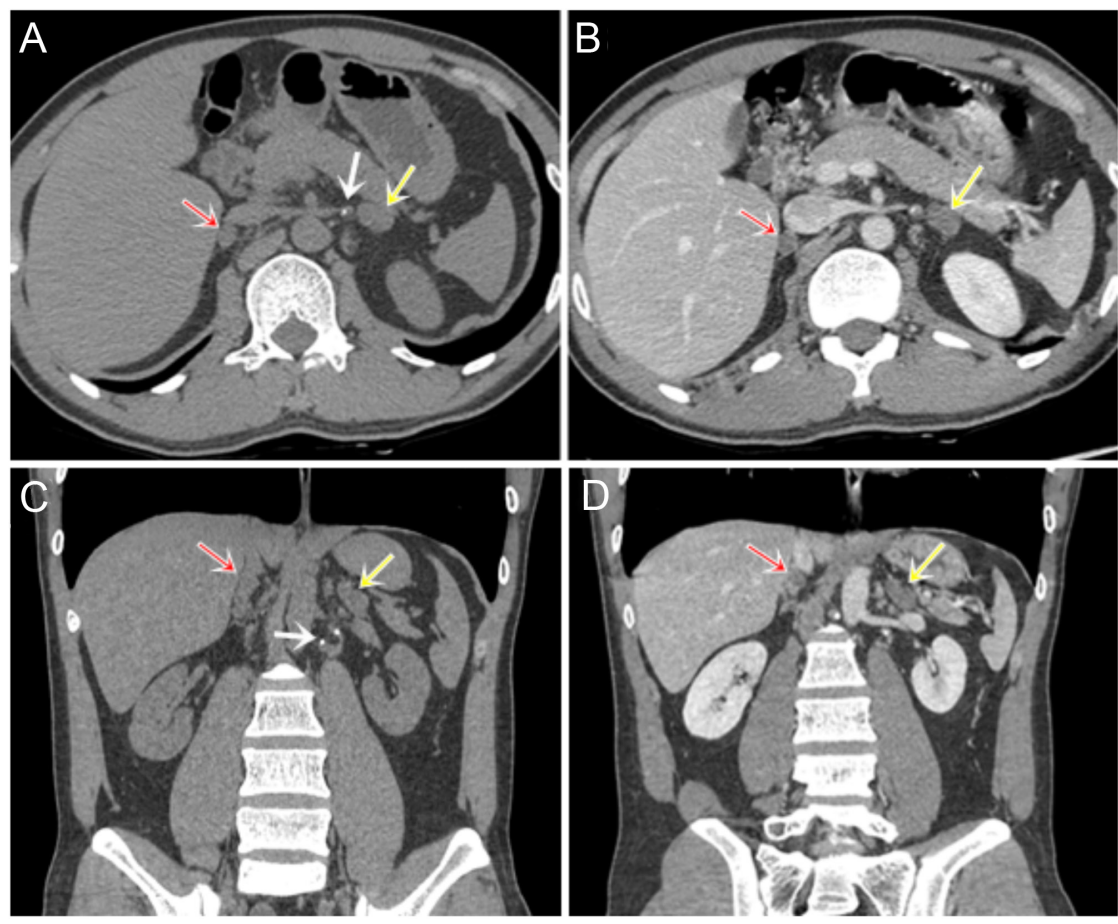

\section{Figure 1}

The unenhanced (axial A and coronal $\mathrm{C}$ reformed image) and contrast-enhanced portal venous phase IV (axial B and coronal D reformed image) revealing a mass-like bilateral adrenal

enlargement (red and white arrows) with a larger mass on the left side, homogeneous low density in the centre (30-40 HU) and with their contours preserved. Calcification of the adjacent lymph nodes was also observed (white arrow).

considered for metastatic adrenal lesions, lymphoma, and infections such as tuberculosis and histoplasmosis.

Neck ultrasound confirmed the presence of bilateral supraclavicular lymph nodes with hilus and fatty infiltration. Thereafter, a biopsy of these suspicious lymph nodes confirmed chronic inflammation. No evidence of pulmonary tuberculosis was found on chest radiography, although chest CT revealed a well-defined 5-mm nodule in the posterior upper lobe of the right lung. Subsequently, bronchoscopy was performed, and PCR using bronchial fluid tested negative for tuberculosis. Additionally, plasma interferon-gamma and human immunodeficiency virus (HIV) serology were negative.

Eventually, an adrenal biopsy was required to establish the differential causes because no extra-adrenal tuberculosis or malignant findings were observed. Therefore, a left retroperitoneal laparoscopic adrenalectomy was performed for diagnostic purposes. During the surgery, an adrenal mass with a nodular lesion adherent to the surrounding tissue was observed. Histopathology of the left adrenal gland revealed granulomatous lesions with central caseous necrosis surrounded by lymphocytes and Langhanstype giant cells which was consistent with tuberculosis inflammation (Fig. 2). A diagnosis of primary adrenal insufficiency caused by bilateral adrenal tuberculosis was established. Hydrocortisone was administered before and after laparoscopic adrenalectomy, with an increased dosage for surgery to prevent acute adrenal insufficiency.

\section{Treatment}

The patient was managed with adrenal hormone replacementincludinghydrocortisoneandfludrocortisone, in addition to 12 months of antituberculous therapy comprising isoniazid, rifampin, ethambutol, and pyrazinamide. The regimen was switched to a combination of rifampicin, isoniazid, and levofloxacin because the

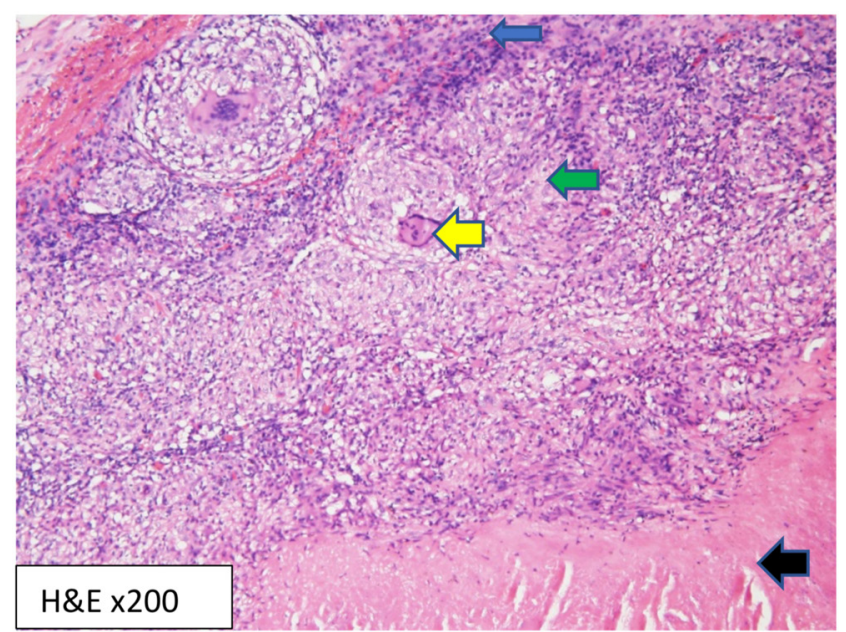

\section{Figure 2}

Histopathology of the left adrenal showing granulomatous inflammation and central caseous necrosis (black arrow) surrounded by lymphocytes and Langhans-type giant cells (yellow arrow). Epithelioid cells (green arrow). Dense lymphocytic cell infiltrate (blue arrow). 
patient was allergic to pyrazinamide and a blurry vision was caused by ethambutol.

\section{Outcome and follow-up}

The symptoms of primary adrenal insufficiency gradually subsided after the initiation of the above medications. The patient gained $8 \mathrm{~kg}$ during the treatment. He continued to use both hydrocortisone and fludrocortisone. During the follow-up, plasma sodium and potassium were stable within the normal range. Plasma aldosterone and renin were $0.97 \mathrm{ng} / \mathrm{dL}$ and $24.7 \mathrm{pg} / \mathrm{mL}$, respectively.

\section{Discussion}

This case report addressed the crucial steps in the diagnosis of bilateral adrenal masses secondary to isolated adrenal tuberculosis, resulting in primary adrenal insufficiency. The patient initially presented with gastrointestinal symptoms, and an incidental finding of bilateral adrenal masses was observed on the CT scan. Manifestations of primary adrenal insufficiency including fatigue, weight loss, and skin hyperpigmentation were noted. No extraadrenal tuberculosis (lymph nodes, lungs, and colon) was determined. Subsequently, the left adrenal mass was surgically removed for establishing a diagnosis. Histopathological examination revealed granulomatous inflammation and caseous necrosis. Therefore, a combination of primary adrenal insufficiency features, CT findings, and histopathology of the adrenal mass helped to establish a definitive diagnosis of bilateral tuberculous adrenalitis, and antituberculous therapy had been administered $(4,6)$.

Nonspecific signs and symptoms of primary adrenal insufficiency, which can masquerade as other diseases such as gastrointestinal diseases and psychological disorders, usually hinder the early diagnosis of this condition. These are the common manifestations of adrenal tuberculosis. Bleicken et al. reported a $68 \%$ incorrect initial diagnosis before the confirmation of adrenal tuberculosis. Gastrointestinal disorders were commonly misdiagnosed as primary adrenal insufficiency, which was also observed in our case. Sometimes, patients do not seek medical attention at the onset of vague features of weakness, tiredness, or anorexia until they are hospitalised with acute adrenal crisis (7).

Morning plasma cortisol concentrations $<83$ $\mathrm{nmol} / \mathrm{L}$ (or $3 \mu \mathrm{g} / \mathrm{dL}$ ) and elevated plasma ACTH levels are hallmarks of primary adrenal insufficiency. The plasma aldosterone level was within the reference range; however, the plasma renin concentration was inappropriately increased, probably as a result of sodium wasting due to mineralocorticoid deficiency $(1,3)$. As a significantly low morning plasma cortisol level of $68.4 \mathrm{nmol} / \mathrm{L}$ was indicative of adrenal insufficiency, a short cosyntropin stimulation test was not required for adrenal insufficiency $(3,8)$.

An adrenal CT scan will provide useful information for the differential diagnosis of primary adrenal insufficiency. In patients with a definitive diagnosis of primary adrenal insufficiency, the aetiologies of bilateral adrenal masses could have certain possibilities such as tuberculosis, fungal infection, lymphoma, and metastasis to the adrenals (1). Most cases of tuberculous adrenalitis ( $>80 \%$ ) have bilaterally enlarged adrenal glands $(9,10)$. These characteristics may result from the hematogenous or lymphatic spread of tuberculous bacilli from primary infection in the lungs. Generally, in the early and active stages of tuberculosis, adrenal glands are enlarged, while long-term disease will have small, atrophic adrenals, and calcification because the adrenal tissue undergoes degeneration and gets completely replaced with fibrous tissue (10).

Diagnosis of tuberculous adrenalitis resulting in primary adrenal insufficiency may not require adrenal biopsy if the following findings are observed: bilateral enlarged adrenal mass on the CT scan accompanied by the evidence of active extra-adrenal tuberculous diseases, especially in areas with a high burden of tuberculosis (6). However, our patient had no evidence of extra-adrenal tuberculous diseases; therefore, an adrenal biopsy was indicated to establish a diagnosis. Fine needle aspiration of the adrenal mass can be performed to achieve sufficient tissue samples for analysis if the size of the mass is large enough, usually $>4 \mathrm{~cm}$ (11). In view of his left adrenal mass measuring only $2 \mathrm{~cm}$, left retroperitoneal adrenalectomy was performed for histopathological examination. Our patient belonged to an endemic region of tuberculosis in Vietnam. Adrenal histopathology revealing granuloma and central caseous necrosis in our patient was considered as tuberculous adrenalitis before the initiation of antituberculous chemotherapy.

In our case, the other causes of primary adrenal insufficiency with bilateral adrenal masses had low probability. Fungal infections of adrenal glands usually occur as the results of disseminated infections in immunocompromised individuals. Endemic area of coccidioidomycosis infection that can affect adrenal gland is only found in South American countries (12). Additionally, both clinical and investigations did not show any suggestions of disseminated fungal infection and HIV, 
therefore histoplasmosis and cytomegalovirus infection were less likely culprits. In male patients with primary adrenal insufficiency, adrenal congenital hyperplasia and adrenal leukodystrophy may be considered if the adrenal glands are normal or small on adrenal imaging (13). However, in our case, bilateral adrenal masses and no family history of adrenal leukodystrophy made these causes unlikely.

Previous studies showed that adrenal glands can be affected in patients with active extra-adrenal tuberculosis. Laway et al. found that the adrenal glands enlargement could be caused by granulomatous adrenalitis in active pulmonary tuberculosis. These patients had lower basal and stimulated plasma cortisol levels than healthy controls. Cortisol levels and the adrenal enlargement were both normalised after tuberculosis treatment (14). In the early stage of tuberculous adrenalitis, $75 \%$ of patients with Addison's disease in the first 2 years had bilateral adrenal enlargement (15). Thereafter, atrophy and calcification of adrenal glands represented an inactive infection of tuberculosis. Olkers suggested that in patients with primary adrenal insufficiency due to tuberculosis, the enlargement of adrenal glands indicates active infection and may require antituberculous drugs (3).

The primary adrenal insufficiency manifests when more than $90 \%$ of the bilateral adrenal glands have been destroyed by tuberculous lesions. Therefore, the adrenal function in our case could not be recovered after an antituberculous treatment and the replacement of glucocorticoid and mineralocorticoid should be lifelong (6).

In conclusion, our case report illustrated a step-bystep approach for establishing a diagnosis of bilateral adrenal masses due to tuberculosis during which a combination of clinical judgement, biochemical and hormonal investigations, radiological examinations, and histopathological evaluation is required.

\section{Patient's perspective}

His health has improved since the initiation of hormonal replacement and antituberculous therapy. The complaints of gastrointestinal disturbance caused a great deal of stress on his life and overall health. He was seriously concerned with the bilateral adrenal masses observed on the abdominal $\mathrm{CT}$, which he initially interpreted as malignancy. He understood the uncertainty in establishing a diagnosis and the need to undergo surgery for diagnostic purposes. His quality of life was significantly better after being placed on hormonal replacement and antituberculous therapy.

\section{Declaration of interest}

The authors declare that there is no conflict of interest that could be perceived as prejudicing the impartiality of the research reported.

\section{Funding}

This work did not receive any specific grant from any funding agency in the public, commercial, or not-for-profit sector.

\section{Patient consent}

Written informed consent has been obtained from the patient for publication of this article and accompanying images.

\section{Author contribution statement}

Thang Viet Tran was the primary physician of the patient. Thang Viet Tran and Nam Quang Tran managed the patient. Chien Cong Phan reported CT scan results and Thao Thi Phuong Doan reported histopathology of adrenal lession. Nam Quang Tran wrote the manuscript and all authors approved the final version of the manuscript.

\section{References}

1 Charmandari E, Nicolaides NC \& Chrousos GP. Adrenal insufficiency. Lancet 2014383 2152-2167. (https://doi.org/10.1016/S01406736(13)61684-0)

2 Barthel A, Benker G, Berens K, Diederich S, Manfras B, Gruber M, Kanczkowski W, Kline G, Kamvissi-Lorenz V, Hahner S, et al. An update on Addison's disease. Experimental and Clinical Endocrinology and Diabetes 2019127 165-175. (https://doi.org/10.1055/a-0804-2715)

3 Oelkers W. Adrenal insufficiency. New England Journal of Medicine 1996 335 1206-1212. (https://doi.org/10.1056/NEJM199610173351607)

4 Soedarso MA, Nugroho KH \& Dewi KAM. A case report: Addison disease caused by adrenal tuberculosis. Urology Case Reports 201820 12-14. (https://doi.org/10.1016/j.eucr.2018.05.015)

5 Lam KY \& Lo CY. A critical examination of adrenal tuberculosis and a 28-year autopsy experience of active tuberculosis. Clinical Endocrinology 200154 633-639. (https://doi.org/10.1046/j.13652265.2001.01266.x)

6 Upadhyay J, Sudhindra P, Abraham G \& Trivedi N. Tuberculosis of the adrenal gland: a case report and review of the literature of infections of the adrenal gland. International Journal of Endocrinology 20142014 876037. (https://doi.org/10.1155/2014/876037)

7 Bleicken B, Hahner S, Ventz M \& Quinkler M. Delayed diagnosis of adrenal insufficiency is common: a cross-sectional study in 216 patients. American Journal of the Medical Sciences 2010339 525-531. (https://doi.org/10.1097/MAJ.0b013e3181db6b7a)

8 Yamamoto T. Latent adrenal insufficiency: concept, clues to detection, and diagnosis. Endocrine Practice 201824 746-755. (https://doi. org/10.4158/EP-2018-0114)

9 Guo YK, Yang ZG, Li Y, Ma ES, Deng YP, Min PQ, Yin LL, Hu J, Zhang XC \& Chen TW. Addison's disease due to adrenal tuberculosis: contrast-enhanced CT features and clinical duration correlation. European Journal of Radiology 200762 126-131. (https://doi. org/10.1016/j.ejrad.2006.11.025)

10 Huang YC, Tang YL, Zhang XM, Zeng NL, Li R \& Chen TW. Evaluation of primary adrenal insufficiency secondary to tuberculous adrenalitis with computed tomography and magnetic resonance imaging: current status. World Journal of Radiology 20157 336-342. (https://doi. org/10.4329/wjr.v7.i10.336)

11 Arambewela M, Ross R, Pirzada O \& Balasubramanian SP. Tuberculosis as a differential for bilateral adrenal masses in the UK. BMJ Case Reports 201912 e228532. (https://doi.org/10.1136/bcr-2018-228532)

12 Paolo Jr WF \& Nosanchuk JD. Adrenal infections. International Journal of Infectiuos Diseases 200610 343-353. (https://doi.org/10.1016/j. ijid.2005.08.001) 
13 Herndon J, Nadeau AM, Davidge-Pitts CJ, Young WF \& Bancos I. Primary adrenal insufficiency due to bilateral infiltrative disease. Endocrine 201862 721-728. (https://doi.org/10.1007/s12020018-1737-7)

14 Laway BA, Khan I, Shah BA, Choh NA, Bhat MA \& Shah ZA. Pattern of adrenal morphology and function in pulmonary tuberculosis: response to treatment with antitubercular therapy. Clinical Endocrinology 201379 321-325. (https://doi.org/10.1111/cen.12170)

15 Sun ZH, Nomura K, Toraya S, Ujihara M, Horiba N, Suda T, Tsushima T, Demura H \& Kono A. Clinical significance of adrenal computed tomography in Addison's disease. Endocrinologia Japonica 199239 563-569. (https://doi.org/10.1507/endocrj1954.39.563)

Received in final form 26 September 2021

Accepted 26 November 2021 